\title{
GPS/Acoustic seafloor geodetic observation: method of data analysis and its application
}

\author{
Masayuki Fujita ${ }^{1}$, Tadashi Ishikawa ${ }^{1}$, Masashi Mochizuki ${ }^{2}$, Mariko Sato ${ }^{1}$, Shin-ichi Toyama ${ }^{1}$, Masato Katayama $^{1 *}$, \\ Koji Kawai $^{1}$, Yoshihiro Matsumoto ${ }^{1}$, Tetsuichiro Yabuki ${ }^{1}$, Akira Asada ${ }^{2}$, and Oscar L. Colombo ${ }^{3}$ \\ ${ }^{1}$ Hydrographic and Oceanographic Department, Japan Coast Guard, 5-3-1 Tsukiji, Chuo-ku, Tokyo 104-0045, Japan \\ ${ }^{2}$ Institute of Industrial Science, University of Tokyo, 4-6-1 Komaba, Meguro-ku, Tokyo 153-8505, Japan \\ ${ }^{3}$ G.E.S.T./NASA Goddard Space Flight Center, Code 926, Greenbelt, Maryland 20771, USA
}

(Received July 21, 2005; Revised September 12, 2005; Accepted September 21, 2005; Online published March 10, 2006)

\begin{abstract}
We have been developing a system for detecting seafloor crustal movement by combining kinematic GPS and acoustic ranging techniques. A linear inversion method is adopted to determine the position of seafloor stations from coordinates of a moving survey vessel and measured travel times of acoustic waves in seawater. The positioning accuracy is substantially improved by estimating the temporal variation of the acoustic velocity structure. We apply our method to the ranging data acquired at the seafloor reference point, MYGI, located off Miyagi Prefecture, in northeast Japan, where a huge earthquake is expected to occur in the near future. A time series of horizontal coordinates of MYGI obtained from seven campaign observations for the period 2002-2005 exhibits a linear trend with a scattering rms of about $2 \mathrm{~cm}$. A linear fit to the time series gives an intraplate crustal velocity of more than several centimeters per year towards the WNW, which implies strong interplate coupling around this region. The precision of each campaign solution was examined at MYGI and other seafloor reference points along the Nankai Trough through comparison of independent one-day subset solutions within the campaign. The resultant repeatability looks to be well-correlated with the temporal and spatial stability of the acoustic velocity structure in the seawater depending on the region as well as the season.
\end{abstract}

Key words: GPS/Acoustic, seafloor geodetic observation, linear inversion, Off Miyagi, intraplate deformation.

\section{Introduction}

Japan is located in a tectonically active region where multiple tectonic plates interact with each other. This causes frequent big earthquakes in and around the country. Among others, a substantial number of huge events have occurred along the undersea plate boundary region, especially on the Pacific side, such as the Tonankai (M7.9 in 1944) and Nankai (M8.0 in 1946) earthquakes. Many researches have been devoted to investigate the interplate coupling on the rupture area for the purpose of elucidating the physical mechanism of such earthquakes. In particular, the backslip distribution on the rupture area of huge earthquakes has been estimated, based on such geodetic data as intraplate crustal velocities measured by the contemporary dense GPS network maintained by the Geographical Survey Institute of Japan (Sagiya, 1999; Suwa et al., 2004). In these studies, however, the lack of data in the marine region limits the resolution and reliability of the back-slip estimation on the undersea plate boundary.

The Hydrographic and Oceanographic Department of Japan (JHOD) has been developing a system for precise seafloor geodetic observation with the GPS/Acoustic com-

*Now at National Astronomical Observatory, 2-21-1 Osawa, Mitaka, Tokyo 181-8588, Japan.

Copyright (c) The Society of Geomagnetism and Earth, Planetary and Space Sciences (SGEPSS); The Seismological Society of Japan; The Volcanological Society of Japan; The Geodetic Society of Japan; The Japanese Society for Planetary Sciences; TERRAPUB. bination technique, using a survey vessel in close collaboration with the Institute of Industrial Science, University of Tokyo (Asada and Yabuki, 2001; Mochizuki et al., 2003, 2005; Fujita, 2003). The primary purpose of our observations is to detect and monitor the crustal deformation caused by the subduction of the oceanic plate near the plate boundary where huge earthquakes repeatedly occur. The JHOD has so far deployed more than fifteen seafloor reference points on the landward slope of the major trenches around Japan, such as the Japan Trench and Nankai Trough, and has begun to obtain successful results (Fujita et al., 2004a).

The idea of the GPS/Acoustic combination technique to measure seafloor crustal movement dates back to the early work done by scientists at the Scripps Institution of Oceanography (Spiess, 1985), examining various methods for undersea positioning with acoustic waves for geodetic purposes. Since then, they, among others, have been making continuous efforts to improve the GPS/Acoustic observation technique (Spiess and Hildebrand, 1995; Spiess et al., 1998; Chadwell et al., 1998, 2002; Chadwell, 2003), using primarily the seafloor reference site on the Juan de Fuca plate off the Canadian coast. Most recently, Gagnon et al. (2005) have discussed intraplate deformation in the South American plate, determined from 100-120 hours of continuous measurements made in 2001 and 2003, with a positioning precision of better than $10 \mathrm{~mm}$.

In Japan, several groups have been trying to develop the GPS/Acoustic system and discussing positioning accu- 


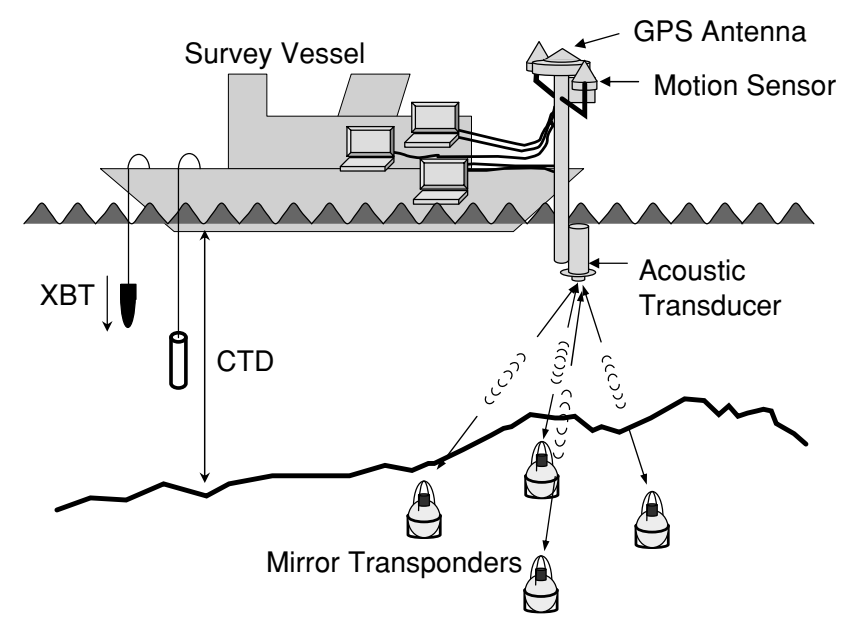

Fig. 1. Schematic picture of the GPS/Acoustic seafloor geodetic observation system.

racy through error estimations. Obana et al. (2000) presented preliminary results from experiments at Sagami Bay, which show an accuracy of measurement on the order of 10 cm. Yamada et al. (2002) estimated measurement errors in acoustic positioning through numerical simulations. Osada et al. (2003) reported results of an experiment carried out off Hawaii Island focusing on the temporal variation of acoustic velocity. Recently, they reported to have detected crustal movement at their reference points: co-seismic displacement at Kumano Basin, in south Japan, caused by the earthquakes SE off Kii Peninsula (M6.9, M7.4) which occurred in 2004 (Tadokoro et al., 2005) and secular movement off Miyagi Prefecture, in northeast Japan (Funakoshi et al., 2005). A theoretical work on the methodology of data analysis was done by $\mathrm{Xu}$ et al. (2005), which presented a mathematical formulation to avoid effects of errors included in the acoustic velocity structure, using single and doubledifference calculation.

The primary purpose of this paper is to describe the methodology of data analysis that we are currently adopting, in particular, the linear inversion method to determine the position of seafloor stations from the kinematic GPS and acoustic ranging results. Then we present our results of analysis with this method obtained from the observed data at the seafloor reference point situated off Miyagi Prefecture, in northeast Japan, where huge earthquakes have repeated with a relatively regular interval of 30 to 40 years. We also discuss the precision of our technique in relation with observation conditions, such as the stability of the undersea acoustic velocity structure.

\section{Measurement System and Campaign Observa- tion}

A schematic picture of the seafloor geodetic observation system that we have developed is shown in Fig. 1. This system consists of a seafloor unit with four (sometimes three) acoustic mirror-type transponders, and an on-board unit with a GPS antenna and an undersea transducer installed on the rigid observation pole of about $8 \mathrm{~m}$ in length, to which it is also attached a dynamic motion sensor. Four transponders on the seafloor are placed to form a square, with a length of the diagonal approximately equal to the water depth in the area.

The system measures ranges from the on-board transducer to the seafloor acoustic transponders through roundtrip acoustic travel times in-between, while simultaneously determining coordinates of the on-board transducer, transferred from those of the GPS antenna, with the attitude of the observation pole measured with the dynamic motion sensor taken into account.

The seafloor transponder functions as a signal retransmitter that receives a ranging signal from the on-board transducer and then transmits the signal back. The ranging signal is a $10 \mathrm{kHz}$ acoustic wave, coded with a 9th order maximum length sequence (M-sequence) with $511\left(=2^{9}-1\right)$ bits. One bit of the code consists of four cycles of the carrier wave, thus the length of the whole signal amounts to $204 \mathrm{~ms}$. The acoustic ranging signals transmitted out and received back by the transducer are both recorded in digital form at a sampling frequency of $200 \mathrm{kHz}$. Kinematic GPS data are mostly sampled at $2 \mathrm{~Hz}$. The acoustic wave velocity profile in the seawater, necessary for transforming travel time into range, is obtained from CTD/XCTD and XBT measurements. See also Asada and Yabuki (2001), Mochizuki et al. $(2003,2005)$ for more details on this system.

The onsite measurements with this system are made aboard a survey vessel, on a campaign basis. In each campaign, we usually perform ranging measurements for more than 3-4 days, for 6-7 hours a day, at present.

Due to the noise produced by the vessel, acoustic ranging measurement can only be carried out when the vessel is drifting with the motor clutch disengaged. Consequently, the geographical distribution of the measurement points depends on the natural wind and water flow, and cannot be controlled. We are trying to make it as balanced as possible by moving the vessel during intervals between drift observations. Typical patterns of geographical distribution of measurement points are shown in Fig. 2.

\section{Overview of Data Analysis}

The data analysis consists of three procedures: (1) acoustic wave analysis to obtain the round-trip travel time between the transducer on board and the seafloor transponder from recorded waveforms, (2) kinematic GPS analysis, and (3) a combination of results from (1) and (2) to get the precise seafloor station position.

For the analysis (1), round-trip travel times of measured acoustic waves are determined by cross-correlation between the original ranging signal and the return signal (Asada and Yabuki, 2001; Toyama, 2003). In this method, the onset of the return signal can be identified as a maximum peak in the correlogram, taking advantage of the characteristics of the M-sequence code.

For the analysis (2), positions of the on-board GPS antenna are determined using a kinematic GPS software called IT (for Interferometric Translocation) which was developed for the precise determination of the trajectory of a rover over very long baselines (Colombo and Evans, 1998; Colombo et al., 2000, 2001). In our practice, we estimate errors included in the kinematic result for the vessel by comparing a 

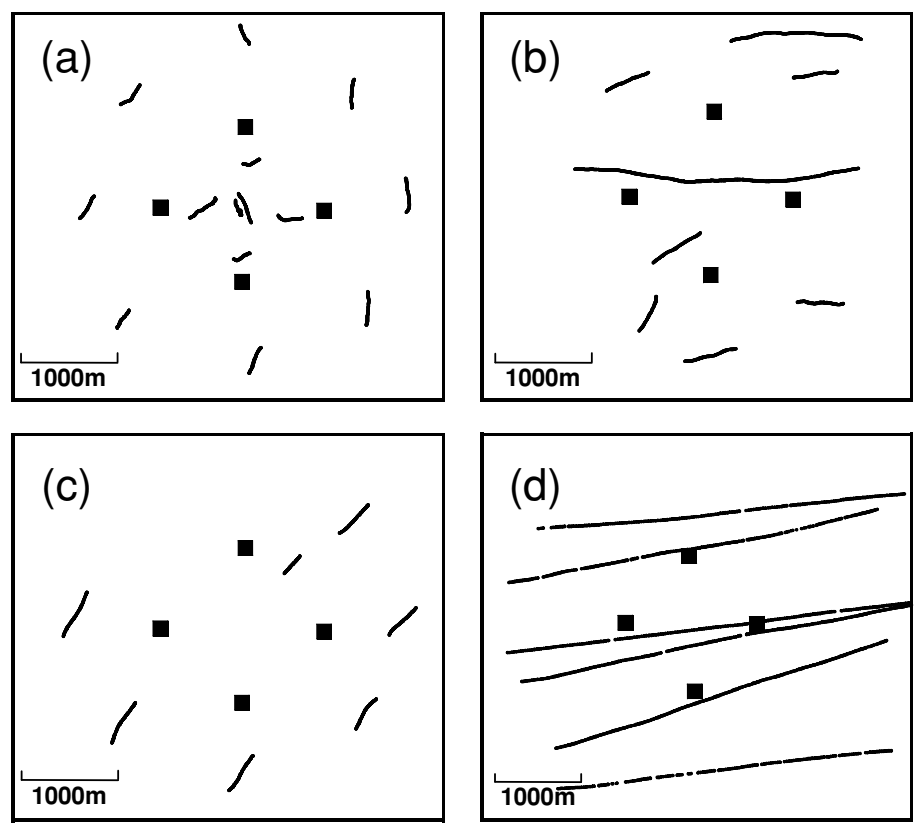

Fig. 2. Typical geographical distributions of acoustic measurement points (wavy lines) in a day around the four seafloor stations (solid squares) characterized by (a) balanced distribution at MYGI on July 15, 2003, (b) irregular flow of current at MYGI on July 14, 2003, (c) poor coverage at MYGI on April 25, 2004, and (d) constant strong flow (due to the Kuroshio current) at SIOW on August 26, 2003.

1-minute average of the GPS-determined sea surface height, with the mean sea surface level, which usually gives a precision of several centimeters; the mean sea surface level for comparison is calculated with a precise local geoid model (Fukuda, 1990), plus a local ocean tide model (Matsumoto et al., 2000), and corrected for the solid earth tide. An example of the comparison between the GPS-measured height and the mean sea surface level is shown in Fig. 3. This error estimation also helps avoid adopting degraded results from bad quality data.

The final analysis (3) is performed by a linear inversion method based on least squares estimation. During this process, we estimate the position of the four transponders on the seafloor individually, but finally take their average as the virtual position of the reference point to be monitored. The detailed methodology of the inversion will be described in the next section.

\section{Method of determining seafloor station position based on the linear inversion formulation}

\subsection{Overview}

In this section, we describe our method of combining results from kinematic GPS and acoustic ranging analyses for determining the position of seafloor stations. Input data are: coordinates of the GPS antenna, round-trip travel time of the acoustic signal, attitude parameters of the vessel (head, roll and pitch), and the acoustic velocity structure in the seawater.

The procedure is as follows: First, using the kinematically estimated coordinates of the GPS antenna at the top end of the observation pole, and the attitude of this pole measured with the motion-sensor, we calculate the position of the submerged acoustic transducer at the other end. And from the successive positions of the transducer and the corresponding round-trip travel times of the acoustic waves,

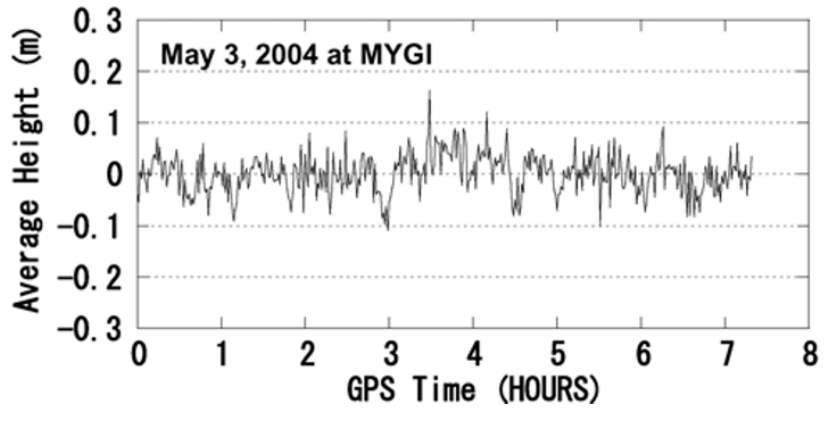

Fig. 3. Example of comparison between the GPS-determined sea surface height and the calculated mean sea surface level for the purpose of estimating errors included in the kinematic result. The time series represents the difference between 1-minute average of the GPS-determined height, on May 3, 2004 at MYGI, and the mean sea surface level, calculated with a precise local geoid model (Fukuda, 1990), plus a local ocean tide model (Matsumoto et al., 2000), and corrected for the solid earth tide.

we estimate the position of the four seafloor mirror stations based on geometrical principles, taking into account the acoustic velocity structure.

In order to estimate the seafloor station positions accurately, it is necessary to have a sufficiently accurate acoustic velocity structure. During our usual operations, we carry out CTD observations (of seawater conductivity, temperature, and depth) with recoverable probes, before and after the ranging measurements of the day, and perform, hourly, similar (XBT and XCTD) measurements, with expendable probes, in between. However, the performances of these instruments are not sufficient. Besides, different empirical equations can be adopted to calculate the acoustic velocity from the observables, that lead to different velocity structures. Moreover, because the velocity varies with time and space, it is practically impossible to make enough observa- 


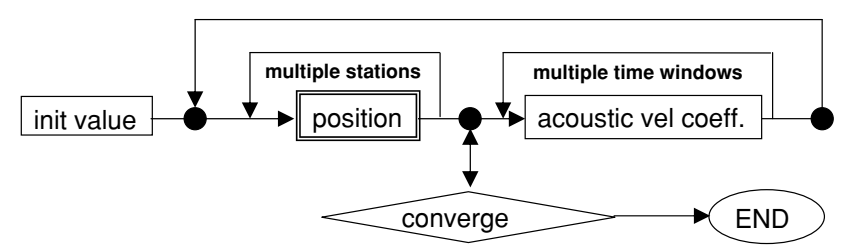

Fig. 4. Simplified algorithm of parameter estimations applied to get the seafloor station position using the round-trip travel time of acoustic waves.

tions to cover all these variations in detail. Thus, for positioning at the centimeter level, it is insufficient to apply a simple, fixed acoustic velocity field obtained from those observations.

On the other hand, the observed travel-time data include not only information on the range, but also that on the velocity structure along the path. Taking advantage of this fact, we estimate corrections to the parameters of the a priori velocity structure. This idea is similar to the estimation of atmospheric delay made as part of the precise GPS data analysis.

For the estimation of the parameters, we apply a theory of Bayesian least squares inversion (Jackson, 1979; Matsu'ura, 1984). Figure 4 exhibits a simplified algorithm of our analysis. First, iterations are made to determine the positions of the multiple stations individually using a certain acoustic velocity structure. Then, using the resultant residuals for the position iterations, an estimation of coefficients for temporal variation of the acoustic velocity structure is made for a given time window. The width of the time window applied for the estimation of the velocity coefficients can be chosen from that of a single observation line, to a maximum of one day. We iterate this process until the position parameters converge.

\subsection{Formulations}

In the following, we describe mathematical formulations used for the estimation of each parameter, i.e., coordinates of an individual seafloor station and/or acoustic velocity coefficients for a certain time window.

First, we introduce general representations of an observation equation and its solution which are common to both parameters. Assuming a linearized relationship between the n-vector of observations $y^{0}$ and the m-vector of model parameters $x$, with an $(n \times m)$ matrix of partial derivatives $A$ and an $n$-vector of Gaussian observation errors $e$, then:

$$
y^{0}=A x+e
$$

When initial estimates of the model parameters and their errors' variance-covariance matrix can be given as a priori information, the model parameter solution $\hat{x}$ can be calculated with the following equation (Jackson, 1979):

$$
\hat{x}=\left(A^{T} E^{-1} A+D^{-1}\right)^{-1}\left(A^{T} E^{-1} y^{0}+D^{-1} x^{0}\right)
$$

or an equivalent form:

$$
\hat{x}=x^{0}+D A^{T}\left(E+A D A^{T}\right)^{-1}\left(y^{0}-A x^{0}\right)
$$

where $x_{0}$ is a vector whose entries are the initial values of the model parameters, $D$ is the a priori variancecovariance matrix for the model parameters, $E$ is the variance-covariance matrix for the observation error $e$, and $A^{T}$ means the transpose of $A$.

The relationship between observation data and model parameters is usually non-linear. However, it can be reasonably assumed that the relationship is linear for small perturbations around the true value of $x$. In Eqs. (1) and (2) or (3), we set $x^{0}=0$, and $y^{0}$ and $x$ are considered to be small corrections to the initial values. The final solution can be obtained iteratively. By introducing the a priori variancecovariance matrix $D$, we can constrain the model parameters, depending on the reliability of the initial value. The simplest application is to have fixed and free parameters.

Next, we describe concrete representations of the observation data and model parameters used for the inversion. As shown in Fig. 4, there are separate observation equations for the position estimation and the velocity estimation.

For the station position estimation, the observation parameters $y^{0}$ are given by:

$$
y^{0}=\left(\begin{array}{llll}
\Delta t_{1(N)} & \Delta t_{2(N)} & \ldots & \Delta t_{n(N)}
\end{array}\right)^{T}
$$

for station ' $N$ '. Parameters on the right hand side are residuals of travel times between observed and calculated from initial positions, arranged as a time series.

The model parameters $x$ are corrections to the initial coordinates of each of the four seafloor mirror stations, expressed as:

$$
x=\left(\begin{array}{lll}
\Delta x_{N} & \Delta y_{N} & \Delta z_{N}
\end{array}\right)^{T}
$$

For the acoustic velocity estimation, the vector of observations $y^{0}$ can be expressed for the time window $\left[t_{k(N)}, t_{l(W)}\right]$, and for the four seafloor mirror stations ' $N$ ', ' $S$ ', ' $E$ ', ' $W$ ' as follows,

$$
y^{0}=\left(\Delta t_{k(N)} \Delta t_{k(S)} \Delta t_{k(E)} \Delta t_{k(W)} \ldots \Delta t_{l(W)}\right)^{T}
$$

As model parameters, we adopted the coefficients of a 2 nd degree polynomial, or quadratic curve, representing the temporal variation of the averaged velocity. The reason for selecting a quadratic as base function is that, in the past, we have succeeded in making reasonable corrections with this function, in an optimum way, by selecting proper lengths for the time windows, which will be described in detail in the next section. The averaged acoustic velocity as a function of time, $V(t)$, is:

$$
V(t)=V\left(t_{0}\right)+a_{0}+a_{1}\left(t-t_{0}\right)+a_{2}\left(t-t_{0}\right)^{2}
$$

where $t_{0}$ represents the reference time, $V\left(t_{0}\right)$ is an initial averaged velocity at time $t_{0}$, and the vector of velocity model parameters, $x$, which are corrections to the quadratic coefficients, is defined as follows:

$$
x=\left(\begin{array}{ccc}
\Delta a_{0} & \Delta a_{1} & \Delta a_{2}
\end{array}\right)^{T}
$$

For obtaining a geometric path of an acoustic wave, the velocity structure is horizontally layered at $200 \mathrm{~m}, 400 \mathrm{~m}$, $800 \mathrm{~m}$ and $1600 \mathrm{~m}$ in depth, where each layer is given a linear variation of velocity with depth, and a ray tracing method is applied. A velocity of every layer varies with time using an identical quadratic curve for a given time window, so that the averaged velocity also varies following the 
Table 1. List of numbers of data for each campaign observation at MYGI used in this study.

\begin{tabular}{ccccccccc}
\hline Epoch & $05 / 2002$ & $10 / 2002$ & $05 / 2003$ & $07 / 2003$ & $05 / 2004$ & $08 / 2004$ & $04 / 2005$ \\
\hline Days & 4 & 2 & 6 & 6 & 8 & 4 & 6 \\
\hline Shots & 3623 & 1306 & 6426 & 7080 & 9280 & 4799 & 7925 \\
\hline
\end{tabular}

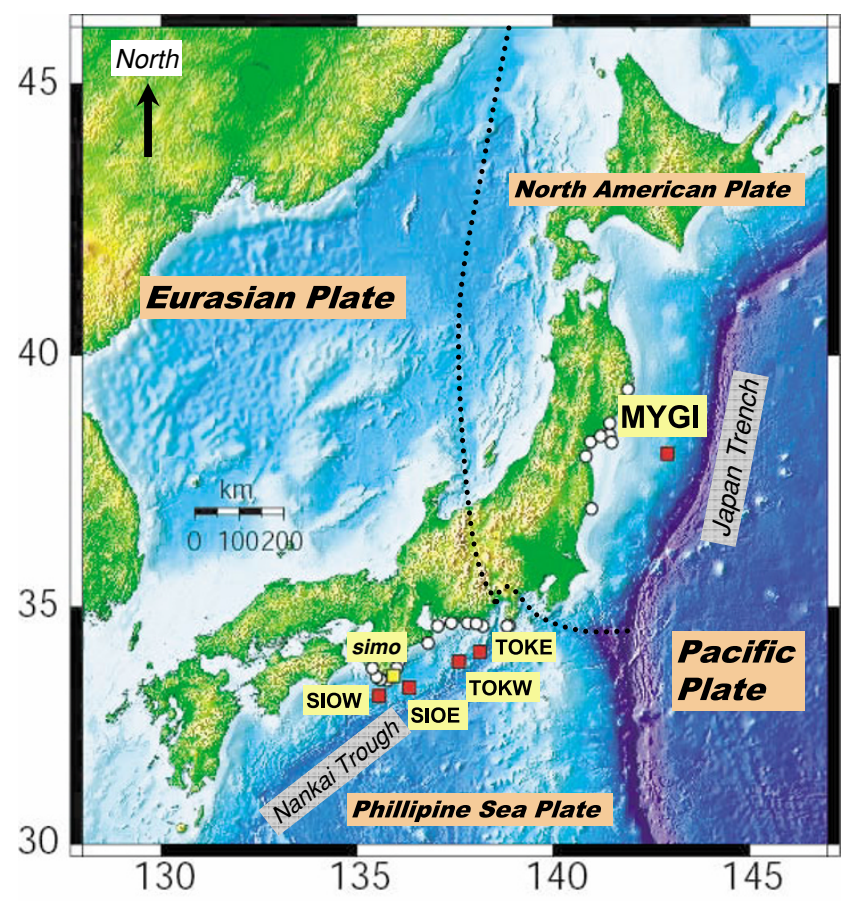

Fig. 5. Locations of the seafloor reference points (red squares) used in this study shown on the topographic map around Japan. Also shown are the position reference of the time series exhibited in Fig. 7, Shimosato, (a yellow square labeled as "simo"), and intermediate land reference stations used for our kinematic GPS analyses (white circles), which are located 50-200 km away from the seafloor reference points.

same curve. In other words, the shape of the vertical profile is assumed to be invariable with time. In reality, this assumption does not hold; for shallower waters it is more variable than for deeper ones. However, numerical examinations show that the positioning result depends little on small fluctuations of the shape of the vertical profile (Sato and Fujita, 2004), so we can safely make this assumption at present. The reason we chose the above number and depths of layering is because it can approximate the observed vertical profile reasonably enough to secure a precision at the centimeter level.

\section{Application to the Observed Data at the Off- Miyagi Reference Point}

We applied the method of analysis described in the previous section to the data obtained at the seafloor reference point off Miyagi Prefecture (labeled as MYGI), in northeast Japan. The location of this point is shown in Fig. 5. MYGI is situated about $100 \mathrm{~km}$ landward from the axis of the Japan Trench, where huge earthquakes have repeated at relatively regular intervals of 30 to 40 years. It is on the eastern edge of the possible rupture area of the next large interplate earthquake. A set of four acoustic transponders

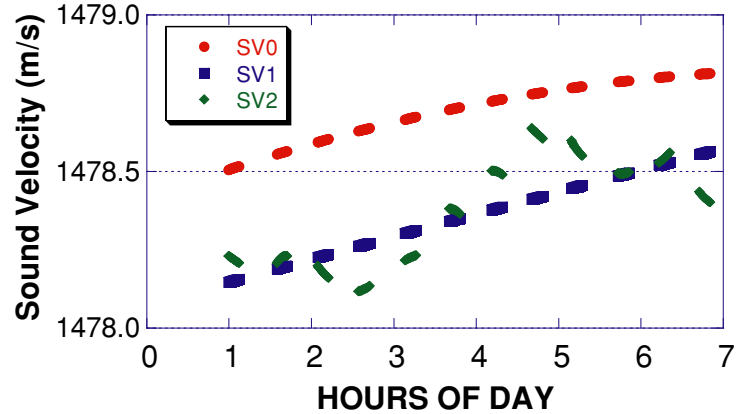

Fig. 6. Representative plots of estimated average acoustic velocity, on April 24, 2004 at MYGI. SV0 (red) represents an initial value given by the 2 nd polynomial fitting to the observed velocities from the CTD, XCTD and XBT, SV1 (blue) is the curve after the daily session estimation as a first step and SV2 (green) is the one estimated for every observation line as a second step. Note that the first step corrects significantly the bias-type errors included in the initial value given by the observations, and the next step deals with irregular short-time variations that cannot be approximated with the 2nd degree polynomial for several hours.

have been installed on the seafloor, at a depth of about 1700 $\mathrm{m}$. The transponders are placed to form a square whose corners are directed to the north, south, east and west. At this particular point, we have been carrying out intensive campaign observations with our system since 2002. Data numbers for the campaigns at MYGI used in this paper are listed in Table 1.

\subsection{Analysis strategy and parameter settings}

5.1.1 Time windows for the acoustic velocity estimations The inversion is done on two consecutive steps, with different time windows for the acoustic velocity estimations. In the first step, the temporal variation of the speed of sound during daily sessions, usually lasting several hours each, is estimated as a 2 nd degree polynomial. In the second step, using the solution from the first step as the initial value, the 2 nd degree polynomial estimation is performed again for every observation line, where each, usually, has been observed for 20-60 minutes.

An example of estimated curves based on this two-step strategy is exhibited in Fig. 6. As shown in this figure, the first step corrects significantly the bias-type errors included in the initial value given by the observations, and the next step deals with irregular short-time variations that cannot be approximated with the 2nd degree polynomial for several hours. It should be emphasized that the second step estimation only, without the first step, cannot deal adequately with bias-type errors, if they are significant.

Fujita et al. (2004b) have shown that the application of this strategy confirms the uniqueness of the final solution of the station position, even if the initial acoustic velocity values used include errors outside the normal performance 

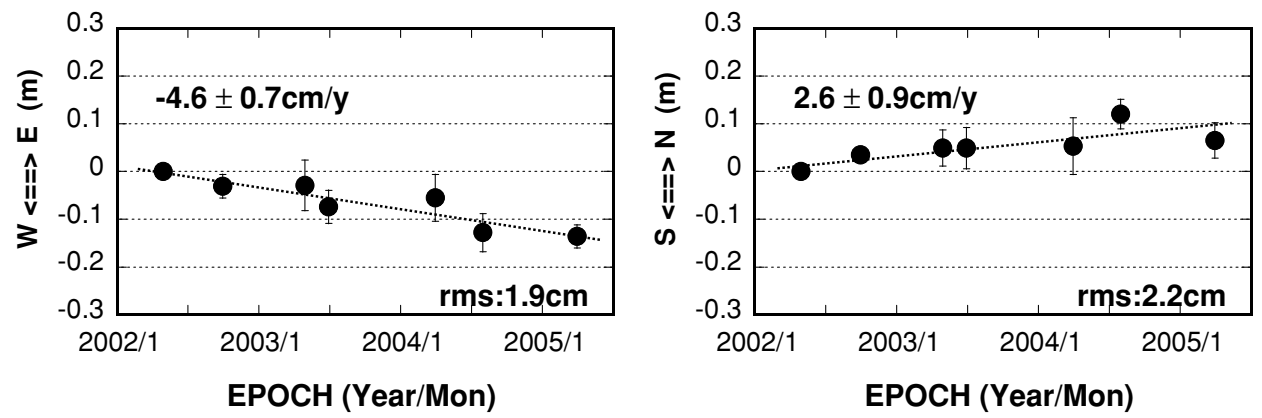

Fig. 7. Time series in the horizontal components obtained at the seafloor reference point MYGI from seven campaign epochs during the period 2002-2005. The left and right panels correspond to the EW and NS components, respectively. The error bar is evaluated according to Eq. (9) in the text, with the coordinates obtained for May 2002 as reference. The root mean square of the scattering around the fitted line is also shown. The position reference is Shimosato, in central Japan.

of the observation instruments.

The initial values for the acoustic velocity structure are based on the CTD, XCTD and XBT measurements. The acoustic velocity is calculated from the observed temperature and conductivity using the empirical equation by Del Grosso (1974). When using XBT data, where only the temperature is measured, the conductivity used is evaluated with a linear fit in time based on the CTD and/or XCTD observations performed on the same day.

5.1.2 Height constraint Our method of analysis can determine in principle the three components of the position of a seafloor station, i.e. two horizontal components and height. Ishikawa and Fujita (2005) proposed that, in order to obtain a horizontal time series as accurately as possible, it is usually very effective to fix the heights of all the epochs to some reasonable value. It summarized the reason as follows.

In an ideal situation, the estimated parameters, which are the three components of position, and the acoustic velocity coefficients, are numerically independent. In some cases, however, the height and undersea acoustic velocity couple with each other to a significant degree because of the geometrical distribution of the ranging data. This would sometimes cause significant errors in the acoustic velocity estimation, which also affects the horizontal position estimation. By constraining the height to some reasonable value, the accuracy in the acoustic velocity estimation would improve, and then the horizontal position accuracy will also improve. It should be noted, however, that this strategy can be applied only when data from many campaign epochs are available, because the reasonable value for the fixed station height has to be found before the final estimation.

In the case of MYGI, it can be reasonably assumed that no significant vertical movement is expected during the campaign period used in this study. In the preliminary 3component analysis, although the determined heights, in two epochs out of seven, deviate by tens of centimeters from the rest, probably because of the errors included in the acoustic velocity estimation, those determined for the remaining five epochs are within ten centimeters repeatability. Ishikawa and Fujita (2005) demonstrated by numerical examinations that a change in fixed height of $\pm 10 \mathrm{~cm}$ affects the estimated horizontal coordinates of the virtual point averaged over four seafloor stations no more than one centimeter. So, we judge that it is reasonable enough to use one of those heights as a fixed value; the value of the epoch May 2002 was chosen in the present analysis.

5.1.3 Weight of the observed data depending on horizontal distance Errors included in the acoustic velocity are to be corrected by the present method as accurately as possible. However, due to limitations in the approximation by the quadratic time function, and also to the effects of spatial inhomogeneity, significant errors may still remain in some cases even after the correction. The effect of errors included in the acoustic velocity is more significant in the longer paths, especially on the horizontal coordinates. Therefore, a reasonable way of decreasing weight for longer paths should help avoid the degradation of the positioning result.

From this viewpoint, we weight the data with $a \cos ^{2} \theta$ law, where $\theta$ is the incident angle of the acoustic ray to the seafloor station. This is applied only in the position estimation; in the acoustic velocity estimation, distant data also play a crucial role and all the data should be weighted equally.

\subsection{Results}

As seen in Table 1, each campaign consists of multiple observation days, and most have more than four. We evaluate a position solution for the campaign epoch using all the multiple-day data acquired. Table 2 lists round-trip travel time residuals for each station and for each campaign analysis. The residuals shown are 70-80 microseconds in average, which corresponds to $5-6 \mathrm{~cm}$ in one-way range.

Figure 7 shows time series of estimated horizontal coordinates of the seafloor stations, from seven campaign epochs at MYGI. The solid circle represents the average of the coordinates of the four mirror stations on the seafloor, relative to the reference campaign epoch, May 2002.

The error bar demonstrates a change of configuration of four stations compared to that of the reference solution. The one-sided value of the error bar, $\sigma$, is evaluated from the following equation:

$\sigma=\sqrt{\sum_{j=1}^{n}\left\{\left(x_{i j}-x_{0 j}\right)-\left(\bar{x}_{i}-\bar{x}_{0}\right)\right\}^{2} / n}\left(\bar{x}_{i}=\sum_{j=1}^{n} x_{i j} / n\right)$

where $x_{0 j}$ and $x_{i j}$ denote coordinates of the $j$ th transponder 
Table 2. Residuals of round-trip travel time for each campaign analysis at MYGI. $N, E, S$ and $W$ denote north, east, south and west stations, respectively.

\begin{tabular}{crrrr}
\hline \multirow{2}{*}{ Epoch } & \multicolumn{4}{c}{ Travel time residuals (microsec) } \\
\cline { 2 - 5 } & \multicolumn{1}{c}{$N$} & \multicolumn{1}{c}{$E$} & \multicolumn{1}{c}{$S$} & \multicolumn{1}{c}{$W$} \\
\hline $05 / 2002$ & 77 & 104 & 80 & 117 \\
$10 / 2002$ & 129 & 109 & 110 & 109 \\
$05 / 2003$ & 97 & 93 & 91 & 91 \\
$07 / 2003$ & 81 & 74 & 81 & 74 \\
$04 / 2004$ & 69 & 75 & 67 & 68 \\
$08 / 2004$ & 76 & 66 & 79 & 72 \\
$04 / 2005$ & 87 & 102 & 79 & 101 \\
\hline
\end{tabular}

for the reference and the $i$ th campaign, respectively, and $n$ is the number of seafloor transponders; in the case of MYGI, $n=4$. It should be noted that the error bar defined here depends on the selection of the reference campaign, so it is not an objective amount in a rigorous sense. However, by carefully choosing a proper reference, it should be a better index for judging the quality of the solution than any other value at present; for instance, a formal error in the least squares inversion does not carry information on the sound velocity errors, whereas the one defined here does, though it is only relative.

The position reference is the Shimosato site, in Wakayama Prefecture, in central Japan, which is one of the ITRF stations equipped also for Satellite Laser Ranging (SLR) observations (Altamimi et al., 2002). We first determine the coordinates of the GPS land reference stations, used for the subsequent differential kinematic GPS positioning of the ship, relative to the precise coordinates of Shimosato through a static analysis. Then, we find the position of the vessel relative to these stations and, therefore, to Shimosato. So, in the end, the locations of the seafloor stations are tied to those of Shimosato via the kinematically determined position of the vessel relative to it, and the acoustically determined position of the underwater stations relative to the vessel.

It must be noted that Shimosato underwent coseismic displacement, amounting to $1.9 \mathrm{~cm}$ with an azimuth of $234^{\circ}$, due to the earthquakes off SE Kii Peninsula (M6.9, M7.4) which occurred in September 2004, revealed by the continuous GPS measurement. The plot of the last epoch in Fig. 7, April 2005, represents the value after the correction with this displacement. Besides, it should also be noted that Shimosato is located on the eastern edge of the Eurasian plate, known to undergo there intraplate deformation at a rate of about $3 \mathrm{~cm} /$ year WNW due to the pressure of the Philippine Sea plate subduction.

The time series shown in Fig. 7 exhibits a linear trend in time with the repeatability of several centimeters. A linear fit to the time series gives a rate of $4.6 \pm 0.7 \mathrm{~cm} /$ year westward and $2.6 \pm 0.9 \mathrm{~cm} /$ year northward. The root mean squares around the fitted line are $1.9 \mathrm{~cm}$ in the EW component, and $2.2 \mathrm{~cm}$ in the NS component. We add the intraplate velocity of Shimosato $\left(3.2 \mathrm{~cm} /\right.$ year, $291^{\circ}$; Sengoku,

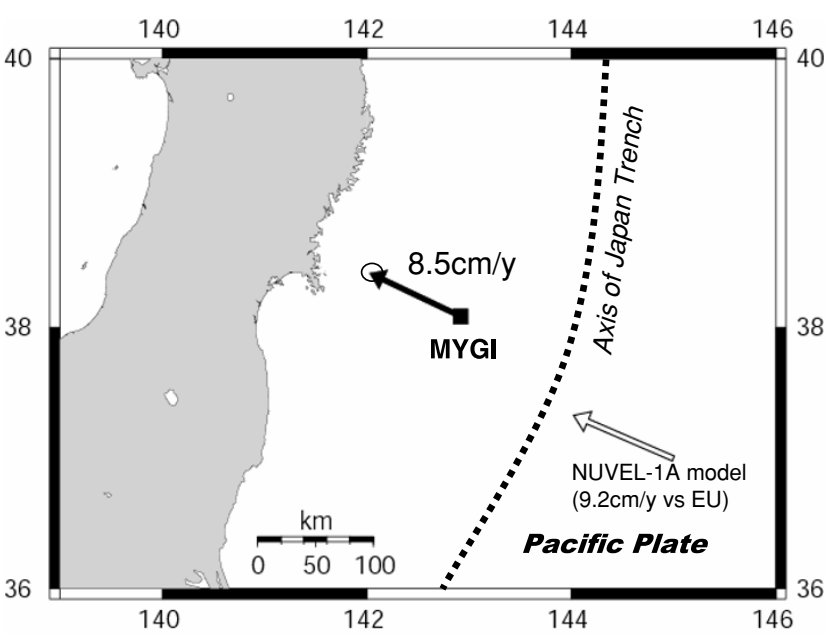

Fig. 8. Crustal velocity vector at MYGI relative to the Eurasian plate evaluated from the time series shown in Fig. 7 after correcting for the intraplate velocity at Shimosato (3.2 cm/year, 291 ; Sengoku, 1998). The ellipse attached to the arrow represents the one-sigma estimation error in the linear fitting to the time series. The velocity of the Pacific plate relative to the Eurasian plate around this region calculated from the plate motion model, NUVEL-1A (DeMets et al., 1994), is also shown with an open arrow.

1998) to the above rate, and obtain $8.5 \mathrm{~cm} /$ year, with an azimuth of $295^{\circ}$, relative to the stable part of the Eurasian continent, which is exhibited with an arrow in Fig. 8. It should be mentioned here that, as shown in Fig. 5, the northeastern part of Japan including MYGI is supposed to be in the North American plate, not in the Eurasian plate, but the relative velocity between both plates is small, so we discuss the crustal velocity vector of MYGI to the Eurasian plate (in Section 6.2).

\section{Discussion \\ 6.1 Repeatability of one-day subset solutions}

In our campaigns, we usually perform ranging measurements for 3, 4, or more days, and evaluate one position solution for the campaign epoch using all the multiple-day data sets so acquired.

To examine the reliability of the campaign solution, we compare independent solutions obtained from the ranging data of each single day. Although one-day worth of data is usually not enough to achieve final precision in many cases, the repeatability of one-day subset solutions is a good index of the precision of the solution for the whole campaign. The subset examination also helps to find bad data or conditions for analysis, to exclude them from the final positioning.

Figure 9 compares the one-day solutions in the horizontal components for four different campaigns at MYGI which have six or more days worth of data. A white square represents the averaged coordinates of four seafloor stations. The leftmost solid plot indicates the campaign solution with all the data, which is shown for comparison. The error bar is evaluated according to Eq. (9); the reference in this case is the leftmost campaign solution.

Repeatability of the subset results is at the several centimeters to 10 centimeters level, when excluding outliers; sometimes a one-day result deviates far from the overall av- 
(a) Apr-May 2003
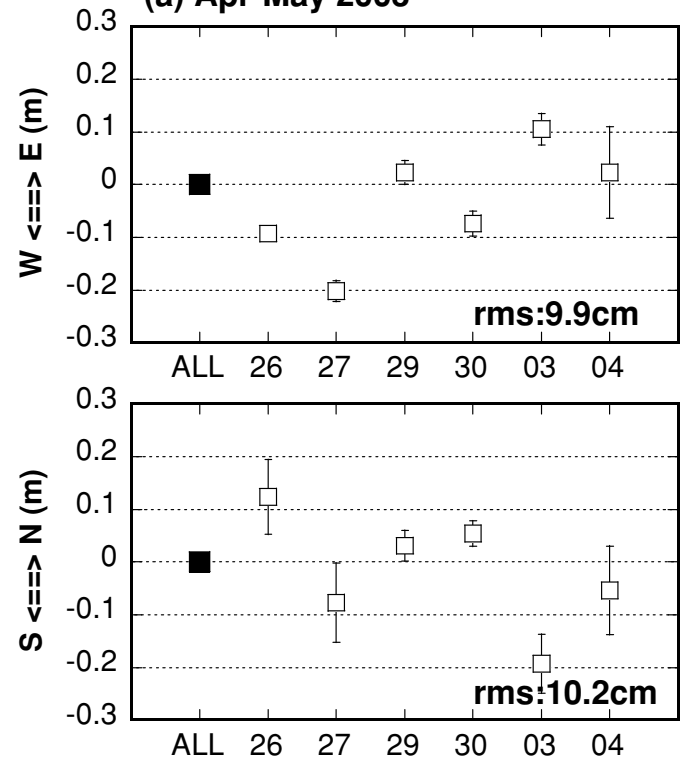

(c) Apr-May 2004
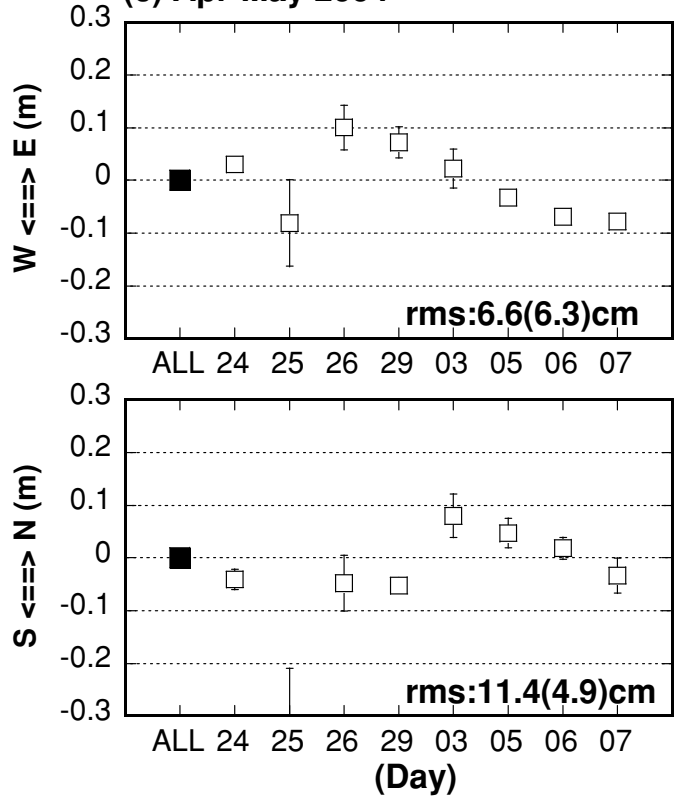

(b) Jul 2003
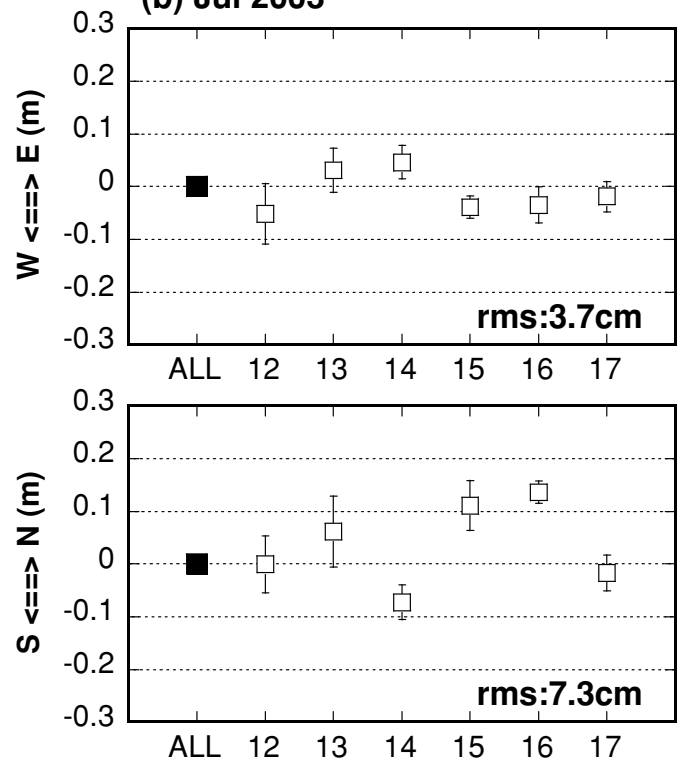

(d) Apr-May 2005
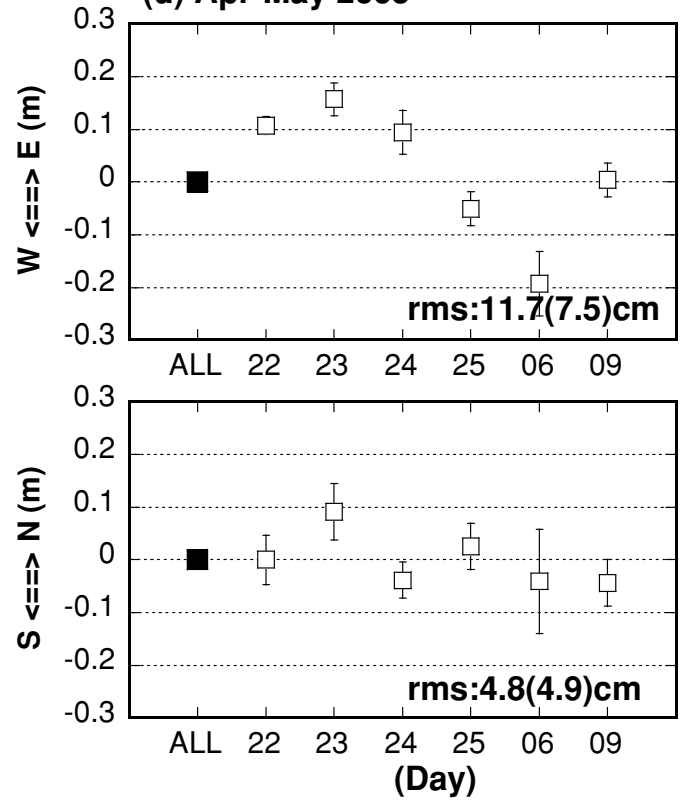

Fig. 9. Comparison of one-day solutions in the horizontal components from four different campaigns at MYGI, each with six or more days' worth of data. The upper and lower panels correspond to the EW and NS components, respectively. A white square represents the average of the coordinates of four seafloor stations. The leftmost plot with a solid square labeled as "ALL" in each panel shows the campaign solution with all the data. The error bar is evaluated with the Eq. (8) in the text, with the leftmost value as a reference. The root mean square of the scattering around the average of one-day solutions is shown in each panel; the value in parenthesis has been evaluated without an outlier.

erage, but this is mainly because the number of data is small or the geographical distribution is very poor for that day, e.g. as shown in Fig. 2(c).

Other than MYGI, we also have examples of one-day subset results for four reference points along the Nankai Trough, TOKE, TOKW, SIOE and SIOW. Locations of these sites are shown in Fig. 5.

Figure 10 shows results from one representative campaign epoch for each site. The applied strategy of analysis is mostly the same as that for MYGI; the only difference is that the height constraint has not been applied for these sites, because we have not yet secured enough campaign epochs for determining the proper heights to be adopted.
Nevertheless, the repeatabilities shown in the figures compare favorably, as a whole, to that of MYGI. One reason for that would be that the epochs shown are in the summer season, when the weather conditions, and the corresponding sea surface conditions, are comparatively good; in the same sense, we found also for MYGI that the summer epochs give better repeatability than those in the spring.

Another reason for the difference may be that the undersea conditions around MYGI are, in general, very complicated compared with those along the Nankai Trough; the area around MYGI is known as the "Perturbed Area", situated in the extensions of both the Kuroshio and Oyashio currents (Kawai, 1972). 
(a) SIOW, Aug-Sept 2003

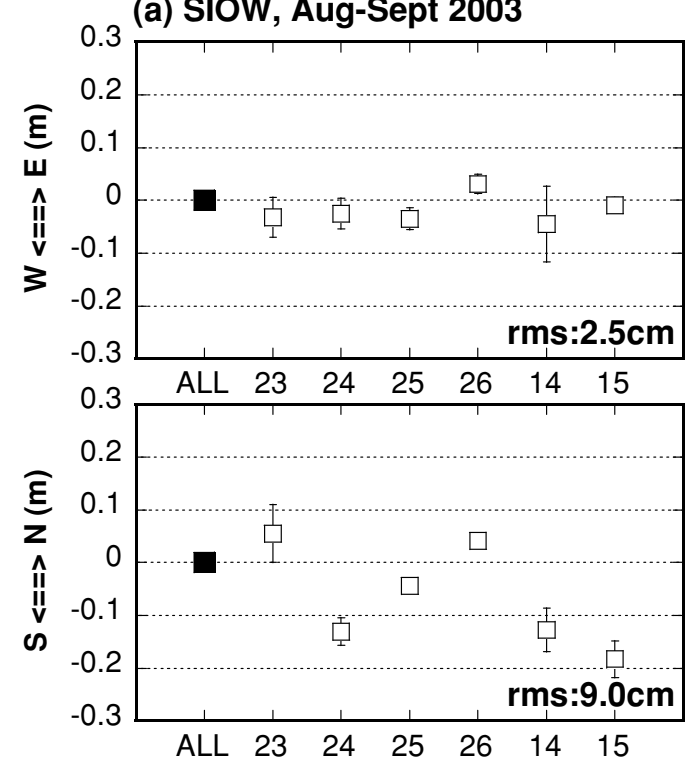

(c) TOKW, Aug 2004
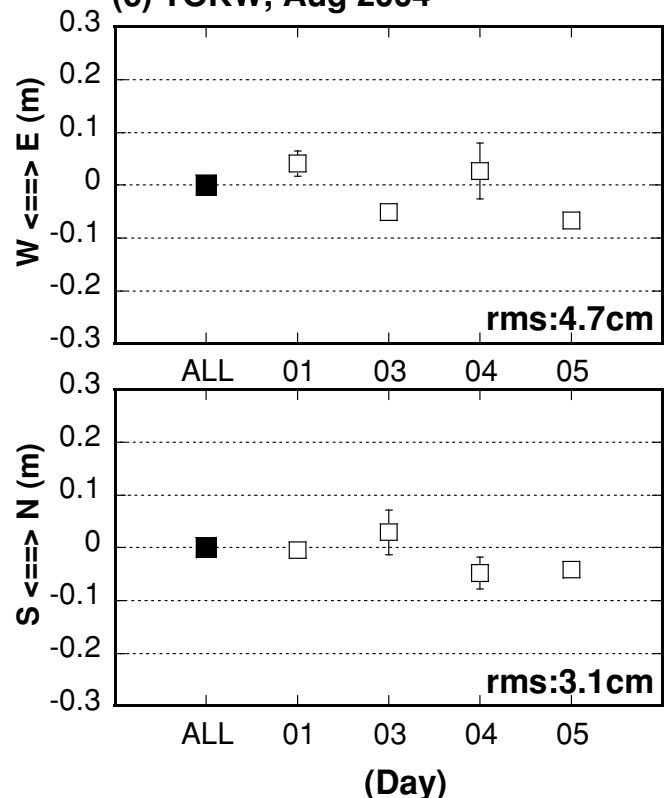

(b) SIOE, Jul 2004
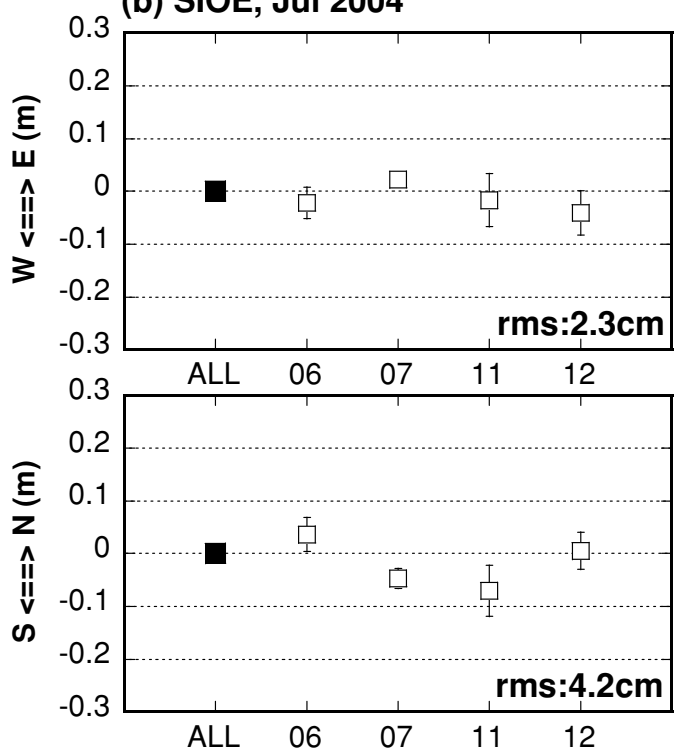

(d) TOKE, Jul-Aug 2004

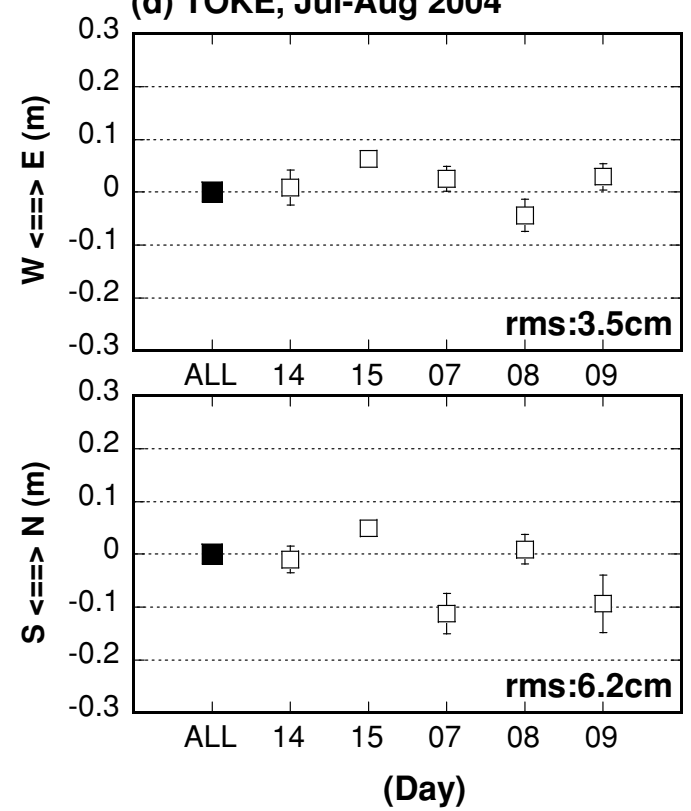

Fig. 10. Same as Fig. 9, but for the four reference points along the Nankai Trough: (a) SIOW in Aug-Sept 2003, (b) SIOE in July 2004, (c) TOKW in Aug 2004 and (d) TOKE in Jul-Aug 2004.

Figure 11 compares the vertical profiles of the seawater temperature, which affects the acoustic velocity more than any other observables, between (a) at MYGI during campaigns in May 2004 and (b) at SIOE in July 2004. As clearly seen, vertical variations in the profiles at MYGI have conspicuous fluctuations on small spatial scales, whereas those at SIOE are very smooth. In addition, there is more disagreement between profiles for different times of day at MYGI, compared to those at SIOE. The variability of the vertical profiles shown in these figures may be a substantial reason why the subset repeatability is worse for MYGI than for that of the other sites shown.

If the height constraint strategy is not applied for MYGI, subset results give worse repeatability than those shown in Fig. 9, especially for the spring season. In other words, the height constraint strategy improves the precision to a sig- nificant degree when the undersea conditions are comparatively unstable.

A closer look at the subset repeatability for the sites along the Nankai Trough tells us that the EW component is better than the NS component except for TOKW. This is especially true for SIOE and SIOW. The reason for this might be that these measurements were carried out basically in the Kuroshio region, when the water flow in the EW direction was very strong. Figure 2(d) is an example of the geographical distribution of the measurement points in this case. Two possible reasons should be pointed out, which may explain the superiority in the EW direction; one is that the geographical distribution of the ranging data is denser, and another is that the undersea velocity structure may be spatially more homogeneous in the direction parallel to the water flow than in the direction perpendicular to it (Yada 

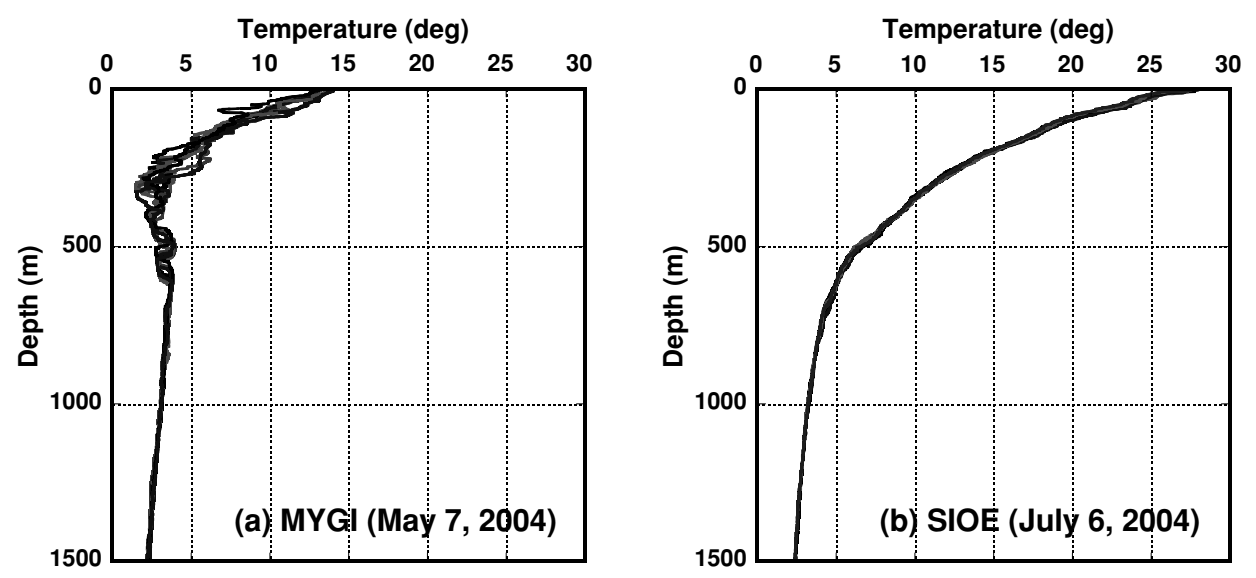

Fig. 11. Vertical profiles of temperature obtained from CTD, XCTD and XBT measurements; (a) at MYGI on May 7, 2004, with nine different epochs of the day superimposed, and (b) at SIOE on July 6, 2004, with eleven epochs.

et al., 2004). The latter would play a major role, because the sparseness of the data distribution does not necessarily degrade the results substantially in many other cases.

6.2 Crustal velocity on the seafloor off Miyagi Prefecture

In northeastern Japan, it is known from GPS static observations at a number of stations, mainly from the GEONET network developed by the Geographical Survey Institute of Japan (Hatanaka et al., 2003), that the crust undergoes intraplate deformations in the WNW direction with velocities of a few centimeters per year. The amount of velocity relative to the Eurasian Plate is about 3-4 cm/year on the Pacific coast of the southern Sanriku region, and decreases inward (Miura et al., 2004).

As seen in the previous section, we have obtained the seafloor movement of MYGI in the WNW direction from the results at seven campaign epochs. The basic trend is consistent with the above movement on land, when we assume that the velocity distribution is extended eastward to the sea area.

The estimated value, $8.5 \mathrm{~cm} /$ year, obtained from the seven epochs, is close to the possible maximum, considering the velocity of the Pacific plate relative to the Eurasian plate along the Japan Trench is $9-10 \mathrm{~cm} /$ year, according to contemporary plate motion models (e.g. NUVEL-1A; DeMets et al., 1994).

Although the estimation error from the linear fit shown in Fig. 7 is about $1 \mathrm{~cm} /$ year, the number of campaign epochs is still not enough to get the statistically stable result. Thus, we need to improve the accuracy of our velocity determination by accumulating further good data from further surveys. However, even if we would allow that errors in the obtained velocity may be a few centimeters per year, it should be concluded, at present, that the seafloor at MYGI moves about twice as fast as those on the coastal area, and also in the WNW direction. This indicates that the interplate coupling within this area is very strong, which is consistent with the results of previous studies (e.g. Suwa et al., 2004).

\section{Summary}

We have been developing a system to determine the precise position of acoustic transponders installed on the seafloor by combining kinematic GPS and acoustic ranging techniques. In this paper, we summarized the methodology of data analysis, in particular, a linear inversion scheme adopted to determine the seafloor station position from coordinates of the moving survey vessel and measured travel times of acoustic waves in seawater. The method includes the estimation of acoustic velocity parameters to correct errors in their values derived from the CTD/XCTD and XBT measurements, and doing this improves the positioning accuracy substantially.

We applied our method to the ranging data acquired at the seafloor reference point located off Miyagi Prefecture, in northeast Japan, where a huge earthquake is expected to occur in the near future. The resulting time series of station positions exhibits a linear trend in the horizontal components indicating an intraplate crustal velocity of more than several centimeters per year towards the WNW. This implies a strong interplate coupling in this region.

The precision of the results was examined by comparing coordinates independently determined from one-day subsets of data from multi-day campaigns. Results from the Off-Miyagi reference point, as well as other four sites along the Nankai Trough, in south Japan, show the repeatability of several centimeters in many cases. Besides, the resultant repeatability looks to be well correlated with the temporal and spatial stability in the acoustic velocity structure in seawater, and depending on the region, as well as on the season.

Over several years, we have substantially improved the accuracy of our technique, which enables us to detect the crustal movement of several centimeters from multiple campaign observations. At present, however, there are still some factors that, both technically and practically, and at different levels, hinder us from getting better results; major issues are (1) tackling the spatial inhomogeneity of the acoustic velocity structure, (2) controlling better the geographical coverage of measurement points, and (3) securing enough measurement time in each campaign. Further efforts are necessary to solve these issues.

Acknowledgments. We thank the Geographical Survey Institute of Japan for providing us with the GEONET GPS data at $1 \mathrm{sec}$ 
sampling for our kinematic GPS analysis. We are indebted to Dr. Zengo Yoshida of Institute of Industrial Science, University of Tokyo, for his sincere support to this project. A lot of staff members of the Hydrographic and Oceanographic Department, including the crew of S/Vs, Meiyo and Kaiyo, have been supporting us for observations and data analyses. Comments from the reviewers, Drs. Hiromi Fujimoto and Katie Gagnon, have improved the manuscipt substantially. Some figures were produced with the GMT software (Wessel and Smith, 1991).

\section{References}

Altamimi, Z., P. Sillard, and C. Boucher, ITRF2000: A new release of the International Terrestrial Reference Frame for earth science applications, J. Geophys. Res., 107(B10), 2214, doi:10.1029/2001JB000561, 2002.

Asada, A. and T. Yabuki, Centimeter-level positioning on the seafloor, Proc. of the Japan Academy, 77, Ser. B, 7-12, 2001.

Chadwell, C. D., Shipboard towers for Global Positioning System antennas, Ocean Engineering, 30, 1467-1487, 2003.

Chadwell, C. D., F. N. Spiess, J. A. Hildebrand, L. E. Young, G. H. Purcell, and H. Dragert, Deep-sea geodesy: Monitoring the ocean floor, GPS World, 9, 44-55, 1998.

Chadwell, C. D., F. N. Spiess, J. A. Hildebrand, and H. Dragert, Seafloor geodetic evidence of episodic spreading $25 \mathrm{~km}$ east of the Juan de Fuca Ridge, EOS. Trans. AGU, 83, Fall Meet. Suppl., Abst., T22A-1130, 2002.

Colombo, O. L. and A. G. Evans, Precise, decimeter-level differential GPS over great distances at Sea and on Land, Proceedings ION GPS-98, Nashville, Tennessee, 1998.

Colombo, O. L., A. G. Evans, M. I. Vigo-Aguiar, J. M. Ferrandiz, and J. J. Benjamin, Long-baseline ( $>1000 \mathrm{~km})$, sub-decimeter kinematic positioning of buoys at sea, with potential application to deep sea studies, Proc. of ION GPS2000, Salt Lake City, U.S.A., 2000.

Colombo, O. L., A. G. Evans, M. Ando, K. Tadokoro, K. Sato, and T. Yamada, Speeding up the estimation of floated ambiguities for subdecimeter kinematic positioning at sea, Proceedings ION GPS-2001, Salt Lake City, Utah, 2001.

Del Grosso, V. A., New equation for the speed of sound in natural water (with comparison to other equations), J. Acoust. Soc. Am., 56(4), 10841091, 1974.

DeMets, C., R. G. Gordon, D. F. Argus, and S. Stein, Effect of recent revisions to the geomagnetic reversal time scale on estimates of current plate motions, Geophys. Res. Lett., 21, 2191-2194, 1994.

Fujita, M., Seafloor geodetic observation-GPS/acoustic combination technique, HydroInternational, 7, 41-43, 2003.

Fujita, M., T. Ishikawa, M. Sato, M. Mochizuki, M. Katayama, S. Toyama, T. Yabuki, A. Asada, and O. L. Colombo, Seafloor geodetic observation along the major trenches around Japan-Focusing on results at offMiyagi area, EOS Trans. AGU, 85(47), Fall Meet. Suppl., Abstract G41A-06, 2004a.

Fujita, M., M. Sato, and T. Yabuki, Development of seafloor positioning software using inverse method, Techn. Rep. Hydrogr. Oceanogr., 22, 5056, 2004b (in Japanese).

Fukuda, Y., Precise determination of local gravity field both the satellite altimeter data and the surface gravity data, Bull. Ocean Res. Inst., Univ. Tokyo, 133 pp, 1990.

Funakoshi, M., H. Fujimoto, A. Sweeney, A. Kuwano, R. Hino, S. Miura, and Y. Osada, GPS/Acoustic submarine positioning using a small buoy in the subduction zone off northeastern Japan, Abstr. Joint Meet. Earth Planet. Sci., J062-001, 2005.

Gagnon, K., C. D. Chadwell, E. Norabuena, Measuring the onset of locking in the Peru-Chile trench with GPS and acoustic measurements, $\mathrm{Na}$ ture, 434, 205-208, 2005.

Hatanaka, Y., T. Iizuka, M. Sawada, A. Yamagiwa, Y. Kikuta, J. M. Johnson, and C. Rocken, Improvement of the Analysis Strategy of GEONET, Bull. Geogr. Surv. Inst., 49, 11-37, 2003.

Ishikawa, T. and M. Fujita, Inverse method and precision improvement for seafloor positioning, Rep. Hydrogr. Oceanogr. Res., 41, 27-34, 2005 (in Japanese with an English abstract).

Jackson, D. D., The use of a priori data to resolve nonuniqueness in linear inversion, Geophys. J. Roy. Astr. Soc., 57, 137-157, 1979.
Kawai, H, Hydrography of the Kuroshio Extension, in Kuroshio-Its Physical Aspects, edited by H. Stommel and K. Yoshida, University of Tokyo Press, 517 pp., 1972.

Matsumoto, K., T. Takanezawa, and M. Ooe, Ocean tide models developed by assimilating TOPEX/POSEIDON altimeter data into hydrodynamical model: a global model and a regional model around Japan, $J$. Oceanogr., 56, 567-581, 2000.

Matsu'ura, M., Bayesian estimation of hypocenter with origin time eliminated, J. Phys. Earth, 32, 469-483, 1984.

Miura, S., T. Sato, A. Hasegawa, Y. Suwa, K. Tachibana, and S. Yui, Strain concentration zone along the volcanic front derived by GPS observations in NE Japan arc, Earth Planets Space, 56, 1347-1355, 2004.

Mochizuki, M., M. Sato, M. Katayama, T. Yabuki, Z. Yoshida, and A. Asada, Construction of seafloor geodetic observation network around Japan, Recent Advances in Marine Science and Technology, 2002, 591600, 2003.

Mochizuki, M., M. Fujita, M. Sato, Z. Yoshida, M. Katayama, T. Yabuki, and A. Asada, Repeated trials of seafloor geodetic observation around Japan, Recent advances in marine science and technology, 2004, 11-18, 2005.

Obana, K., H., Katao, and M. Ando, Seafloor positioning system with GPS-acoustic link for crustal dynamics observation-a preliminary result from experiments in the sea, Earth Planets Space, 52, 415-423, 2000.

Osada, Y., H. Fujimoto, S. Miura, A. Sweeney, T. Kanazawa, S. Nakao, S. Sakai, J. A. Hildebrand, and C. D. Chadwell, Estimation and correction for the effect of sound velocity variation on GPS/Acoustic seafloor positioning: An experiment off Hawaii Island, Earth Planets Space, 55, e17-e20, 2003.

Sagiya, T., Interplate coupling in the Tokai District, Central Japan, deduced from continuous GPS data, Geophys. Res. Lett., 26, 2315-2318, 1999.

Sato, M. and M. Fujita, Effects of sound velocity profiles in the seafloor geodetic observation, Techn. Rep. Hydrogr. Oceanogr., 22, 42-49, 2004 (in Japanese).

Sengoku, A., A plate motion study using Ajisai SLR data, Earth Planets Space, 50, 611-627, 1998.

Spiess, F. N., Suboceanic geodetic measurements, IEEE Trans. Geosci. Remote Sens., 23, 502-510, 1985.

Spiess, F. N. and J. A. Hildebrand, Employing geodesy to study temporal variability at a mid-ocean ridge, EOS Trans. AGU, 76, 451, 455, 1995.

Spiess, F. N., C. D. Chadwell, J. A. Hildebrand, L. E. Young, G. H. Purcell, Jr., and H. Dragert, Precise GPS/Acoustic positioning of seafloor reference points for tectonic studies, Phys. Earth. Planet. Inter., 108, 101-112, 1998.

Suwa, Y., S. Miura, A. Hasegawa, T. Sato, and K. Tachibana, Spatiotemporal change of interplate coupling in the Northeastern Japan subduction zone, J. Seismol. Soc. Jpn., 56, 471-484, 2004 (in Japanese with an English abstract)

Tadokoro, K., R. Ikuta, M. Ando, T. Okuda, S. Sugimoto, K. Takatani, and K. Yada, Repeated observation of sea-floor deformation at Kumano Basin, Japan (2), Abstr. Joint Meet. Earth Planet. Sci., J062-007, 2005.

Toyama, S., Analysis for acoustic data in sea bottom geodetic observation, Techn. Rep. Hydrogr. Oceanogr., 21, 67-72, 2003 (in Japanese).

Wessel, P. and W. H. F. Smith, Free software helps map and display data, EOS Trans. AGU, 72, 441, 445-446, 1991.

Yada, K., R. Ikuta, M. Ando, T. Okuda, K. Tadokoro, M. Kuno, S. Sugimoto, and K. Takatani, Spatial variations in acoustic velocity at Kuroshio region for the accurate ocean-bottom positioning, EOS Trans. $A G U, \mathbf{8 5}(47)$, Fall Meet. Suppl., Abstract G21A-0147, 2004.

Yamada, T., M. Ando, K. Tadokoro, K. Sato, T. Okuda, and K. Oike, Error evaluation in acoustic positioning of a single transponder for seafloor crustal deformation measurements, Earth Planets Space, 54, 871-881, 2002.

$\mathrm{Xu}, \mathrm{P}$, M. Ando, and K. Tadokoro, Precise, three-dimensional seafloor geodetic deformation measurements using difference techniques, Earth Planets Space, 57, 795-808, 2005.

M. Fujita (e-mail: masayuki-fujita@kaiho.mlit.go.jp), T. Ishikawa, M. Mochizuki, M. Sato, S. Toyama, M. Katayama, K. Kawai, Y. Matsumoto, T. Yabuki, A. Asada, and O. L. Colombo 